International Journal of Advanced Biological and Biomedical Research

Available online at http:www.ijabbr.com

Volume 8, Issue 2 (2020) pp. 128-132

DOI: 10.33945/SAMI/IJABBR.2020.2.3

Review Article

\title{
Introduction of Microbial as a Major Factor in the Human Body
}

\section{Seyed Mojtaba Fakhr Hosseini*,1, Saman Aiubi²}

${ }^{1}$ Division Microbiology, Department of Molecular and Cell Biology, Faculty of Basic Sciences, Lahijan branch, Islamic Azad University(IAU), Lahijan, Gilan, Iran

${ }^{2}$ Mycobacteriology Research Centre (MRC), National Research Institute of Tuberculosis and Lung Disease (NRITLD), Shahid Beheshti University of Medical Sciences, Tehran, Iran

*Corresponding Author E-mail: fakhr.hosseini2000@gmail.com

Received: 03 August 2019, Revised: 18 September 2019, Accepted: 08 October 2019

\begin{abstract}
The aim of this study is introducing microbials, symbiotic microorganisms, as an effective agent in the human body. Microbial means the general and universal ecosystem of riches, all genes and masses of micronutrients, regardless of their genera and species. The symbiotic microbials have a symbiotic relationship with host creatures. This means that these microbes can also be partners, both pathogens. For example, all bacteria, fungi and other types of germs that live natively in the human skin are called microbiotics. The largest microbial of the human body is the microbioum of the digestive tract and, in particular, the large intestine. This term, although used more in medicine, is also valid in other branches of microbiology. They play an important role in health and diseases. Trillions of bacteria, called microbials, that make up our bodies in our societies, involve the release of chemicals in the method of digestion, the delivery of medication, and the development and development of diseases such as heart disease, metabolic disorders (metabolism), or even cancer progression.
\end{abstract}

Key words: Microbial, Main factor, Microbium of the lower digestive system, Intestinal microbial

\section{Introduction}

Microbial is a habitat for various species of bacteria and fungi, which, in a symbiosis balanced but unique form, are part of our biological characteristics (Clemente et al., 2012). In an unuasual study, scientists discovered that migrating people from developing countries to developed countries immediately lost their native microbials and quickly gained microbial American-European populations. People living in developing countries have a greater variety of bacteria in their intestines that are suitable for general well being. Studies have shown that Southeast Asian migrants will soon lose this diversity after immigration. Many of those who migrate to the United States are initially healthy, but over time and over again, they develop obesity. This is 
not only due to a diet or a rise in calories, but something else is happening. Rather, researchers believe that this is due to the change in the number of trillions of microscopic living things in our bodies and called microbials (Gibson et al., 2019). Researchers from the University of Minnesota at the laboratory looked at the world of germs that live in the gastrointestinal tract. These invisible creatures are called intestinal microbiosis and are very important for human health. These microbes help digest foods, train immune systems, and fight infections. Changes in the microbiota of the human intestine are associated with any significant chronic human disease (Macfarlane et al., 2007). In fact, information indicates that microbial and its changes can cause many of these diseases, including obesity. The researchers looked at what the problem with the microbiota of the intestine came from as a developing country into the United States (Martínez et al., 2002). In this study the two Hmong Asian peoples were typically surveyed from the mountainous areas of China, Vietnam, Laos and Thailand, and Karen from Myanmar and Thailand (Chowdhury et al., 1996). In this case, the first generation born after the migration of these people to the United States was studied. The research showed that with the migration of the two Hmong and Karen populations to the United States, the diversity of their microbials was reduced to the Americans, and that Asian immigrants had fewer microbials than non-immigrants. These conditions were true for the children of these people, that is, children more than their grandchildren. They had less microbial content. The study looked at two groups of bacteria called Bacteroides and Prevotella, which are specially tailored to be Western and Asian. These two bacteria are not necessarily bad or good, but are dominant in the intestinal microbiosis in different populations around the world (Yousuf et al., 2019). After immigration to the United States, Bacteroides bacteria were replaced by their Asian type. Like other western populations, these communities suffer from obesity. These changes are noticeably more pronounced in children, which can affect their longterm health (Roberts et al., 2019). Previous studies have shown that having the wrong microbes can increase the obesity in the mouse, so these changes can be dangerous. In fact, information indicates that microbial and its changes can cause many of these diseases, including obesity. Many studies have found that our microbials affect almost every aspect of our health; and its microbial composition, which varies from person to person, is very important in any aspect, including weight gain and mood. Some microbial researchers have argued that this change begins with differences in our genes; however, the large-scale study challenges this idea and provides evidence that the link between microbioum and health is even more important than what it is thought about. In fact, this hypothesis states that genetics plays an important role in determining the microbial diversity (microbial symbiosis) among individuals (Wang et al., 2019). The aim of this study is to present and investigate the microbials as an effective agent in the human body.

\section{Human microbial project: drug production from intestinal microorganisms}

Human microbial is the hidden world of our natural vision that has recently been taken into consideration (Chang et al., 2019). Scientists have recently discovered that imbalances in gastrointestinal microorganisms can create many diseases (Arunima et al., 2019). The human digestive system is a complex and precise ecosystem that is endangered by such factors as aging, travel, or even the use of new drugs (Yeoh et al., 2019). 


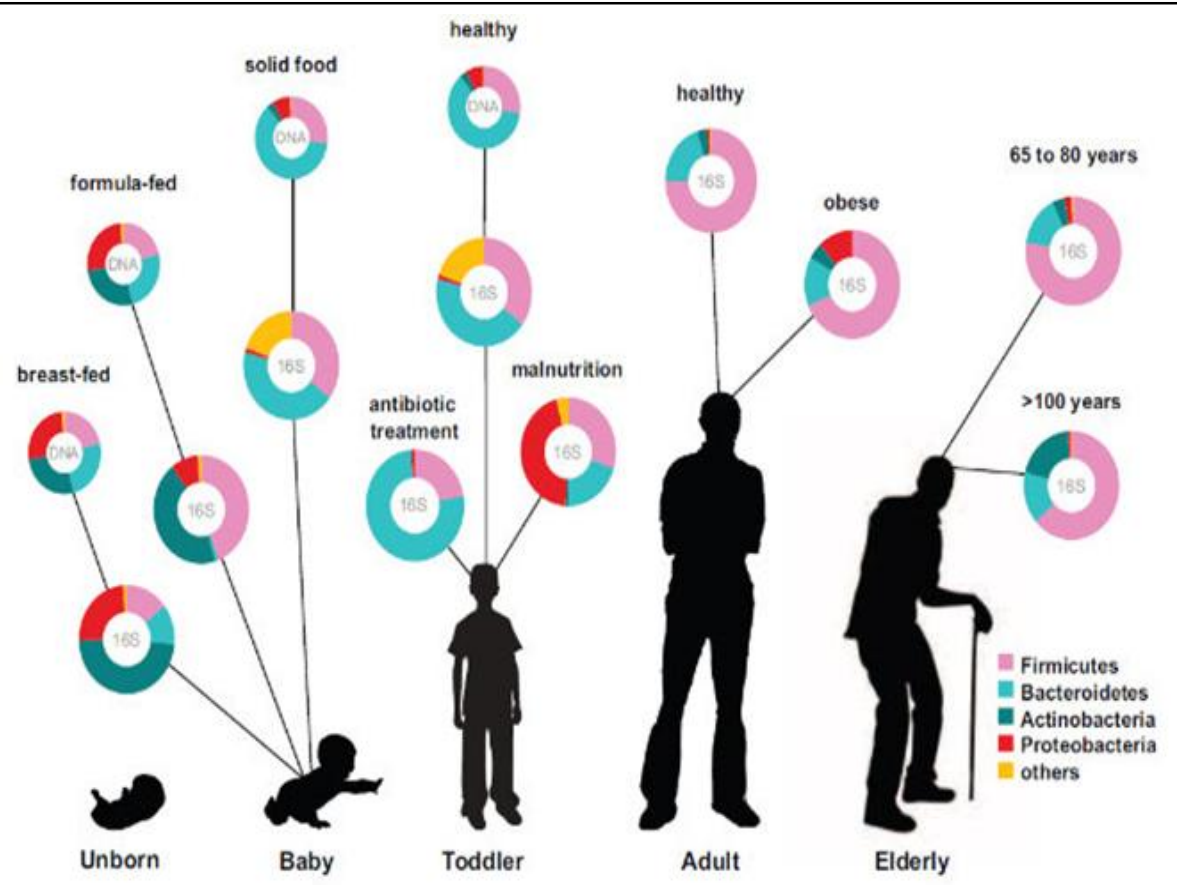

Figure 1. Schematic view of gastrointestinal ecosystems and curing diseases through microbials

A series of human-derived germs are washed and purified with ethanol, and are ultimately packaged in tablet form. Experts use the term "fecal microbiota transplant" or FMT to carry out bowel movements from one person to another, and it is expected that Ceres's oral treatment would have a miraculous effect on the subject. One of the deadliest human intestinal infections caused by the Clostridium difficile bacterium is called "fecal membrane enterocolitica", which is not a result of antibiotic therapy (Huang et al., 2019). This is due to people's losing most of their digestive system bacteria because of the severe use of antibiotics. Early experiments showed that Ceres's treatment eliminated C. Diff infection in $97 \%$ of cases, a result that pharmaceutical companies did not even dream of. The startup requested the approval of the treatment to the FDA and is therefore the first active company in the field of targeted treatment of digestive disorders, from C. diff to Crohn's Disease (Zhao et al., 2019). A few weeks later, the company announced the results of its second phase of the test: an infection of about half of the patients had recurred to C. diff, a statistically significant difference in treatment with Ceres. Perhaps the first stool pill failed. Within a short distance from the main headquarters of Ceres, the world's largest stool bank is called Open Biome (Verran et al., 2019). Scientists at the center also try to treat C. diff infection, with the difference that their product has been used for several years. Open Biome believes in the powerful impact of pure and untreated stools. Each month, hundreds of people volunteer anonymously to their bankers, and experts make a weighing and grading of each sample based on the Bristol Stool Scale. In the next step, the salt solution is added to the contents of the package and the resulting compound is frozen, to be sent to physicians and researchers throughout the United States (Parmar et al., 2019). Since the launch of Open Biome in 2012, this simple and voluntary fecal donation process has managed to cure more than 15,000 cases of C. diff. The importance of delivering medicine to the patient's body is by no means important; enzymes, colonoscopies, oral capsules containing compressed and frozen stools are all successful in treating the disease, but 
reusing the contents of the intestine has become one of the challenges of regulatory bodies. According to FDA experts, and of course common sense, the Open Biome approach will only be a short-term solution, as long as a better way is invented. Although stool donors are evaluated and screened for dangerous illnesses, no trial can guarantee everything.

\section{The role of microbium in reducing heart disease}

Most people know that the risk factors for heart disease include high blood pressure and high cholesterol and smoking. But these factors are not so effective in predicting the risk of heart disease in young people, women and some ethnic groups (Milkov., 2018). One of the ways to assess the risk of a heart attack is to measure the hardness of the arteries. It does not have much to do with high cholesterol or smoking, but it is closely related to inflammation. Inflammation is our natural reaction to injury, but when this reaction is too intense, it can lead to several diseases, including arthritis and asthma. In fact, the more inflammation in the body is, the more likely it is for heart disease and atherosclerosis (Toczyłowska-Mamińska et al., 2018 ). According to a previous study, one of the ways to reduce inflammation in the body is the presence of beneficial bacteria in the intestine. In fact, the level of healthy intestinal microbes is lower in the body of people with diseases such as psoriatic arthritis, diabetes and inflammatory bowel disease caused by inflammation. Recent studies have found that in women with an increased severity of arteries in their bodies, there is less microbial diversity, and women with more healthy veins have more microbial diversity. Additional, the amount of useful compounds produced by germs in the bloodstream of people with more healthy veins is more than that of others. According to the study, approximately $10 \%$ of the stiffness of the vessels is related to the amount of germs and the compounds produced by them. In contrast, less than $2 \%$ of this risk factor is due to high cholesterol, smoking, diabetes and obesity. Unlike genetic hazards, germs in the intestines and materials produced by them can be changed (Wu et al., 2018). One way to do this is to eat beneficial bacteria by eating probiotic foods or supplements. Another way is to eat omega-3s and fibers found in oils and vegetables, whole-hearted breads, a variety of brains, beans and bitter chocolate. A complete report of this research has been published in the European Heart Journal.

\section{The effect of intestinal microbial on autism}

The intestinal microbial has several effects on human health and now it can even alleviate the symptoms of autism based on new studies on microbial health (Preininger et al., 2018). A study on 18 autistic children with severe digestive problems also reported that balancing microbiota in these children could at the same time relieve symptoms of autism and digestive problems, as well as relieve the symptoms of these children over the time. Two years have passed since the review began. Medical experts in the United States said: We treated children with autism by changing the gut microbial (Yeo et al., 2018; Nitschke et al., 2018 ). All children with symptoms of digestive problems, such as diarrhea, constipation, abdominal pain, had a marked improvement in digestive problems and behavioral abnormalities due to changes in the microbiota of the intestine (Chang et al., 2019). According to these experts, two years after these patients were examined, the behavioral symptoms of these children and their digestive problems improved. 


\section{Conclusion}

In the present study, we introduced the role of microbials as an effective agent in the human body. The main point is the careful examination of the mechanisms of germ cell function that relates them to human health. In the meantime, why and how of different effects of different strains of bacteria and their effects on the human body, as well as dietary effects on microbial changes, are more important. It is accordingly necessary to obtain more results from research on animals and to evaluate them in relation to human studies, preferably randomized through clinical trials.

\section{References}

Arunima, A, Das, JK, Suar, M. (2019). Gut Microbes in Liver Diseases: Dietary Intervention for Promoting Hepatic Health. Dietary Interventions in Gastrointestinal Diseases. Elsevier. p: 11731.

Clemente, JC, Ursell, LK, Parfrey, LW, Knight, R. (2012). The impact of the gut microbiota on human health: an integrative view. Cell., 148(6):1258-1270.

Chowdhury, R, Sahu, GK, Das, J. (1996). Stress response in pathogenic bacteria. J. Biosci., 21(2):149-160.

Chang, CP, Hsiao, CT, Fann, WC. (2019). Risk factors associated with bacteremia correlated with mortality in patients with acute bacterial skin and skin structure infection. Intern. Emerg. Med., $14(2): 259-264$.

Chang, I, Cho, GC. (2019). Shear strength behavior and parameters of microbial gellan gumtreated soils: from sand to clay. Acta Geotechnica., 14(2):361-375.

Gibson, GR, Roberfroid, MB. (1995). Dietary modulation of the human colonic microbiota: introducing the concept of prebiotics. J. nutr., 125(6):1401-1412.

Huang, C, Zeng, Y, Luo, X, Ren, Z, Tang, B, Lu, Q, Gao, S, Wang, S, Mai, B. (2019). In Situ Microbial Degradation of PBDEs in Sediments from an E-Waste Site as Revealed by Positive Matrix Factorization and Compound-Specific Stable Carbon Isotope Analysis. Environ. Sci. Technol., 53(4):1928-1936.

Milkov, AV. (2018). Secondary microbial gas. Hydrocarbons, Oils and Lipids: Diversity, Origin, Chemistry and Fate. p: 1-10.

Macfarlane, S, Dillon, J. (2007). Microbial biofilms in the human gastrointestinal tract. J. Appl. Microbiol., 102(5):1187-1196.

Martínez, JL, Baquero, F. (2002). Interactions among strategies associated with bacterial infection: pathogenicity, epidemicity, and antibiotic resistance. Clin. Microbial. Rev., 15(4):647679.

Nitschke, M, Silva, SSE. (2018). Recent food applications of microbial surfactants. Crit. Rev. Food Sci. Nutr., 58(4):631-638. 
Preininger, C, Sauer, U, Bejarano, A, Berninger, T. (2018). Concepts and applications of foliar spray for microbial inoculants. Appl. Microbiol. Biotechnol., 102(17):7265-7282.

Parmar, ND, Shukla, SR. (2019). Decolourization of dye wastewater by microbial methods-A review. Indian J. Chem. Technol. (IJCT)., 25(4):315-323.

Roberts, CA, Buikstra, JE. (2019). Bacterial infections. Ortner's Identification of Pathological Conditions in Human Skeletal Remains: Elsevier. p:321-439.

Toczyłowska-Mamińska, R, Szymona, K, Król, P, Gliniewicz, K, Pielech-Przybylska, K, Kloch, M, Logan, BE.(2018). Evolving microbial communities in cellulose-fed microbial fuel cell. Energies, 11(1):124.

Yousuf, B, Mishra, A. (2019). Exploring Human Bacterial Diversity Toward Prevention of Infectious Disease and Health Promotion. Microb. Diver. Genom. Era, p: 519-533.

Yeoh, YK, Chen, Z, Hui, M, Wong, MC, Ho, WC, Chin, ML, Ng, SC, Chan, FKL, Chan, PKS. (2019). Impact of inter-and intra-individual variation, sample storage and sampling fraction on human stool microbial community profiles. Peer J., 7:e6172.

Yeo, J, Kim, T, Jang, J, Yang, Y. (2018). Practical maximum-power extraction in single microbial fuel cell by effective delivery through power management system. Energies, 11(9):2312.

Verran, J, Redfern, J, Moravej, H, Adebola, Y. (2019). Refreshing the public appetite for 'good bacteria': menus made by microbes. J. Biol. Educat., 53(1):34-46.

Wang, Y, Li, L, Xiong, R, Guo, X, Liu, J. (2019). Effects of aeration on microbes and intestinal bacteria in bioaerosols from the BRT of an indoor wastewater treatment facility. Sci. Total Environ., 648:1453-1461.

Wu, X, Shi, Z, Zou, L, Li, CM, Qiao, Y. (2018). Pectin assisted one-pot synthesis of three dimensional porous $\mathrm{NiO} /$ graphene composite for enhanced bioelectrocatalysis in microbial fuel cells. J. Power Sou., 378:119-124.

Zhao, X, Huang, J, Lu, J, Sun, Y. (2019). Study on the influence of soil microbial community on the long-term heavy metal pollution of different land use types and depth layers in mine. Ecotoxicol. Environ. Safety, 170:218-226.

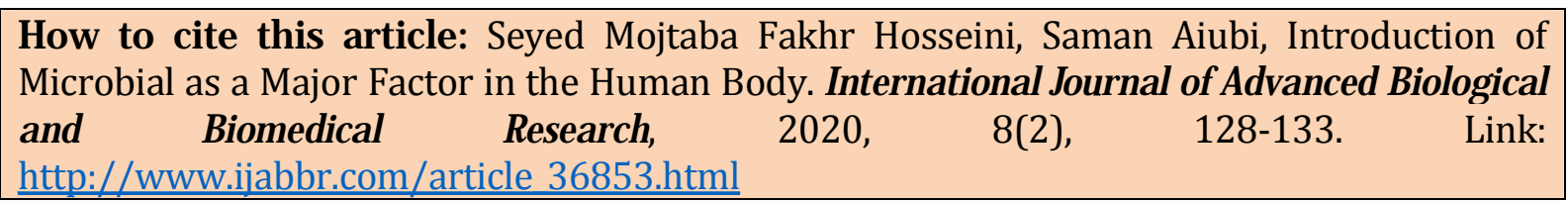

